\title{
Microtubule-mitochondrial attachment determines cell division symmetry and polarity in fission yeast
}

\author{
Leeba Ann Chacko ${ }^{1,2}$ and Vaishnavi Ananthanarayanan ${ }^{1,2} \bowtie$ \\ ${ }^{1}$ Centre for BioSystems Science and Engineering, Indian Institute of Science, Bengaluru, India \\ ${ }^{2}$ Current affiliation: EMBL Australia Node in Single Molecule Science, School of Medical Sciences, University of New South Wales, Sydney, Australia
}

\begin{abstract}
Association with microtubules inhibits the fission of mi- 44 tochondria in Schizosachharomyces pombe. Here we show 45 that this attachment of mitochondria to microtubules is an 46 important cell intrinsic factor in determining division sym- 47 metry as well as maintaining polarity. By comparing mutant 48 cells that exhibited enhanced attachment and no attachment 49 of mitochondria to microtubules (Dnm1 $\Delta$ and Mmb1 $\Delta$ re- 50 spectively), we show that microtubules in these mutants dis- 51 played aberrant dynamics compared to wild-type cells, which 52 resulted in errors in nuclear positioning. This translated to 53 cell division asymmetry in a significant proportion of both 54 Dnm1 $\Delta$ and Mmb1 $\Delta$ cells. So too, microtubule pivoting 55 was enhanced in both mitochondrial mutants, resulting in a 56 fraction of the cells in these populations displaying polarity 57 defects. The asymmetric division in Dnm1 $\Delta$ and Mmb1 $\Delta{ }_{58}$ cells resulted in unequal distribution of mitochondria, with 59 the daughter cell that received more mitochondria growing 60 faster than the other daughter. Taken together, we show the 61 existence of homeostatic feedback controls between mito- 62 chondria and microtubules in fission yeast, which directly in- 63 fluence mitochondrial partitioning and thereby, cell growth. 64 tioning

Microtubules; mitochondria; cell division; polarity; mitochondrial parti-

Correspondence: vaish@unsw.edu.au
\end{abstract}

\section{Introduction}

Symmetric cell division is the hallmark of most eukaryotic 70 cells. Fission yeast (Schizosaccharomyces pombe) is a rod- 71 shaped, unicellular eukaryote that divides symmetrically dur- 72 ing mitosis (1). A single cell grows by polarised tip exten- 73 sion from about $7 \mu \mathrm{m}$ to $14 \mu \mathrm{m}$ in length. Once the cell has 74 grown to $14 \mu \mathrm{m}$ in length, cells cease to grow and proceed 75 to divide by assembling an actomyosin contractile ring at the 76 geometrical centre of the cell $(2,3)$. Subsequently, the two 77 daughter cells formed post mitosis are of equal length. Due 78 to their ability to divide medially and produce identically- 79 sized daughter cells, fission yeast is a powerful tool in cell 80 cycle research.

81

One of the key players involved in ensuring symmetric 82 division in fission yeast has been identified to be the micro- ${ }_{83}$ tubule (MT) cytoskeleton (4). A typical fission yeast cell 84 contains an average of three to five MT bundles that emanate 85 from nucleators embedded in the nuclear envelope, and are 86 positioned along the long axis of the cell (5). The pushing 87 forces of the individual bundles against the cell periphery in an interphase cell ensure the medial placement of the nucleus (4). This medial placement enables positioning of the division plane at the centre of the cell (6). As a result, attenuating the dynamics of MTs causes severe cell division defects. Similarly, polarised growth in $S$. pombe is enabled by the deposition of growth factors at cell tips by kinesin-like motor proteins and MT +TIP proteins $(7,8)$. Perturbation of MT dynamics therefore results in polarity defects.

Contrary to their depiction in textbooks, mitochondria are not discrete, static entities, but rather a network of tubules that are in an equilibrium between fission and fusion. This balance between fission and fusion is essential for proper mitochondrial function, with dysfunction being associated with several cellular metabolic defects (9). The dynamin-related GTPase Drp1 (Dnm1 in yeast) is the major mitochondrial fission protein, whereas two sets of GTPases Mfn1/2 and Opa1 bring about fusion of the outer membrane and inner membrane of the mitochondria respectively (10-12). Dnm1 is cytosolic but assembles as rings around the mitochondrial outer membrane and undergoes GTP hydrolysis to effect constriction and eventual scission of mitochondria $(13,14)$. In the absence of Dnm1, mitochondria exist as a single, long network that spans the entire cell, but remains attached to the MT (15).

In fission yeast, mitochondria are also bound to the MTs via the linker protein Mmb1 (16). In recent work, we showed that the absence of Mmb1 results in mitochondrial fragmentation due to the inability of Dnm1 to assemble around mitochondria bound to MTs (17). In cells with shorter MTs than normal, we observed several shorter mitochondria, whereas in cells with longer MTs than wild-type (WT), we observed fewer, longer mitochondria. Importantly, the total mitochondrial volume between the WT cells and mutant strains with shorter or longer MTs was conserved, indicating that the predominant result of altered MT dynamics was a change in mitochondrial morphology. We therefore established a causal link between MT dynamics and mitochondrial morphology (17).

Here, we explored the outcome of altered mitochondrial form, and thereby their attachment to MTs in context of cell division and polarity. We observed that both Dnm1 $\Delta$ and $\mathrm{Mmb} 1 \Delta$ cells displayed increased asymmetric cell division, as well as polarity defects. We set out to investigate the mech- 
A

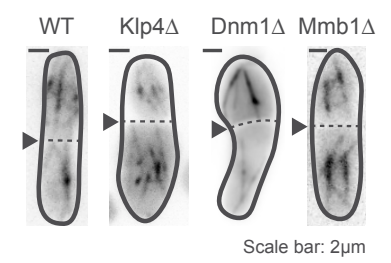

B

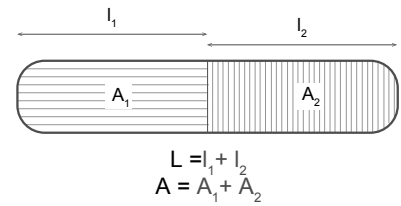

D

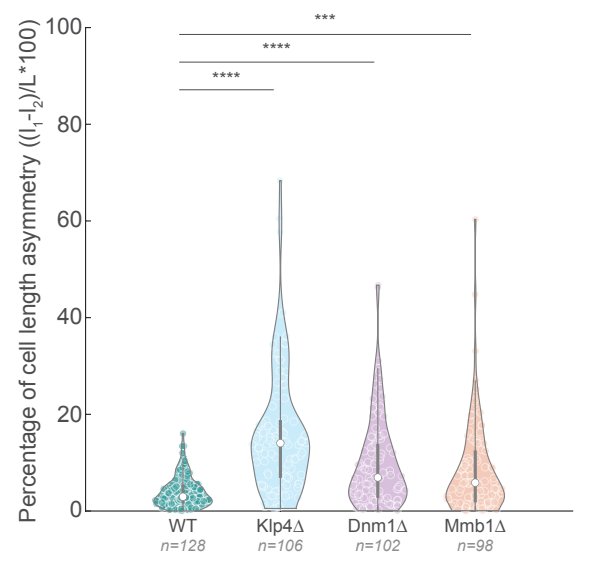

C

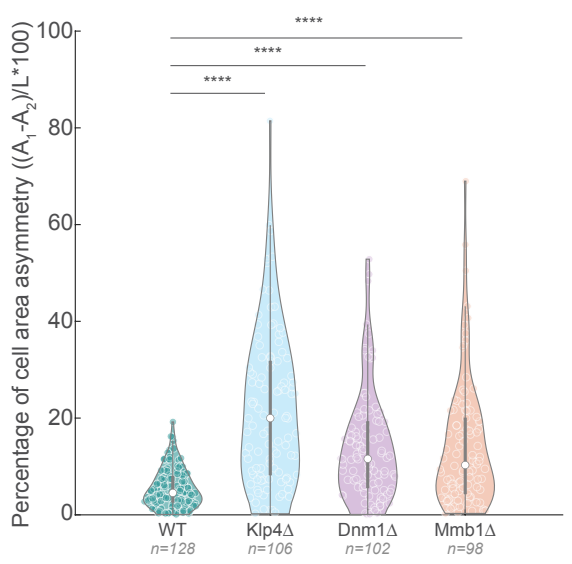

$\mathrm{E}$

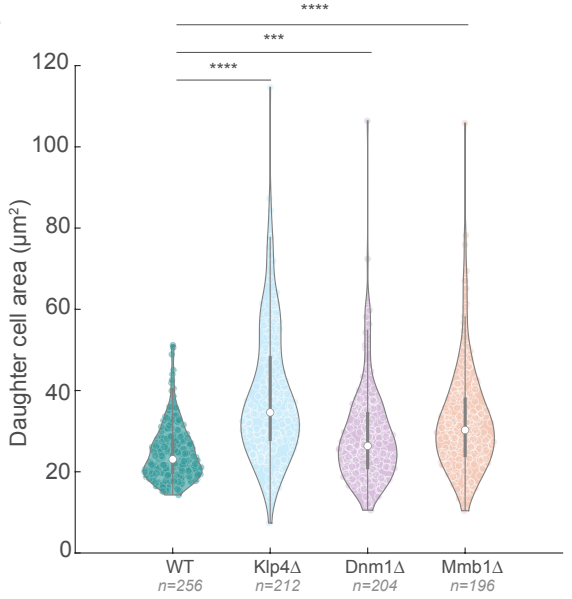

Fig. 1. Dnm1 $\Delta$ and Mmb1 $\Delta$ cells exhibit asymmetric cell division. A, Maximum intensity projected images of MTs in WT, Klp4 $\Delta$, Dnm1 $\Delta$ and Mmb1 $\Delta$ (strains L972, FY7143, KI001, G5B, Dnm1 $\Delta$ and VA069, see Table S1), with the cell division plane (dashed line) indicated with the black arrowheads. B, Schematic of the method employed to measure cell length and area asymmetries. C, Plot of asymmetry in cell lengths between the daughter cells in WT, Klp4 $\Delta$, Dnm1 $\Delta$ and Mmb1 $\Delta$ cells. D, Plot of asymmetry in cell areas between the daughter cells in WT, Klp4 $\Delta$, Dnm1 $\Delta$ and Mmb1 $\Delta$ cells. E, Plot of daughter cell area in WT, Klp4 $\Delta$, Dnm1 $\Delta$ and Mmb1 $\Delta$ cells. In C, D and E, the asterisks represent significance $\left(^{\star * \star *}=\mathrm{p}<10^{-4}\right.$ and ${ }^{\star \star *}=\mathrm{p}<2 \times 10^{-4}$ respectively), Kruskal-Wallis test for non-parametric data.

anism by which alteration of mitochondrial form resulted in 108 these cellular homeostasis defects.

\section{Results}

A significant proportion of Dnm1 $\Delta$ and Mmb1 $\Delta$ cells ${ }^{112}$ divide asymmetrically. Cells lacking the mitochondrial fission protein Dnm1 contain a single long mitochondrial network ((15), Fig S1A). This long mitochondrion was attached to MTs along the length of the cell, such that when MTs were depolymerised using MBC (methyl-2-benzimidazolecarbamate), we observed recoiling of the mitochondrial network (Fig. S1B, Video S1). This evinced that there was an enhanced attachment of mitochondria to MTs in Dnm1 $\Delta_{120}$ cells. On the other hand, cells lacking the mitochondria-MT ${ }_{121}$ linker protein Mmb1 do not associate with MTs (16). In 122 our previous work, we showed that this dissociation of mi- 123 tochondria from MTs results in fragmentation of the mito- ${ }^{224}$ chondrial network (Fig. S1A, (17)). When we followed di- 125 viding Dnm1 $\Delta$ and Mmb1 $\Delta$ cells, we observed that $\sim 15 \% 126$ of these cells exhibited asymmetry in both cell length and ${ }_{127}$ cell area during division, compared to $\sim 5 \%$ in WT cells ${ }_{128}$
(Fig. 1). Accordingly, the daughter cells in Dnm1 $\Delta$ and Mmb1 $\Delta$ background were also distributed across a larger range of areas than the WT cells (Fig. 1E). This high degree of asymmetry during division is similar to the phenotype in Klp4 $\Delta$ (MT-stabilising kinesin-like protein (18), Fig. 1), Pom $1 \Delta$ (polarity-determining protein kinase (19), Fig. S1C, D) which are known to exhibit asymmetry in division as well as cell polarity defects. Cells lacking the heteromeric kinesin-8 Klp5/6 have longer MTs and mitochondria than WT (17), and therefore also have increased attachment of mitochondria to MTs. Klp5/6 $\Delta$ cells also showed increased asymmetric division compared to WT cells (Fig. S1C, D).

We asked if the asymmetry could have arisen due to defects in mitochondrial function in the mutant cells. To answer this question, we quantified the proportion of asymmetry in dividing $r h o^{0}$ cells. $r h o^{0}$ cells lack mtDNA, relying primarily on glycolysis for ATP production, and therefore grow slower on fermentable carbon sources (20). We did not observe significant differences in cell division asymmetry between WT and $r h o^{0}$ cells (Fig. S1C, D). Mitochondrial form is also linked to reactive oxygen species (ROS) levels, 
A

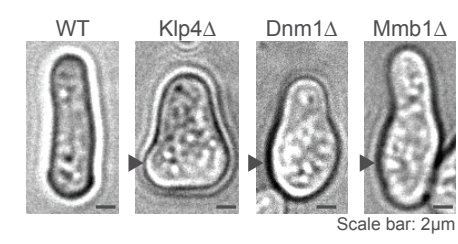

B

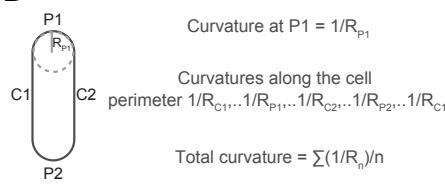

C

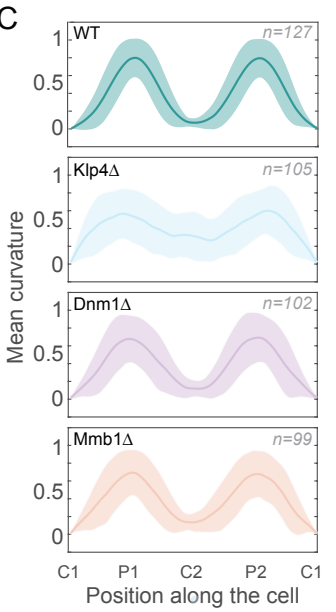

D

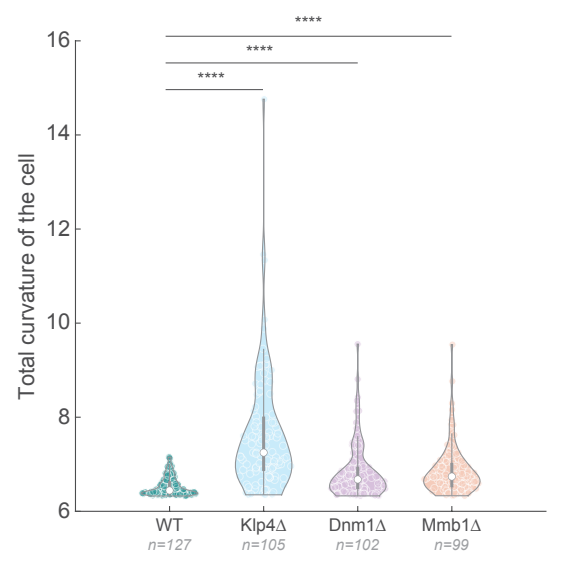

Fig. 2. Dnm1 $\Delta$ and Mmb1 $\Delta$ cells exhibit cell polarity defects. A, Bright-field images of representative WT, Klp4 $\Delta$, Dnm1 $\Delta$ and Mmb1 $\Delta$ cells (strains L972, FY7143, KI001, G5B, Dnm1 $\Delta$ and VA069, see Table S1). The arrowheads point to polarity defects. B, Schematic describing the method used to quantify curvature of the cell. C, Plot of mean curvature (solid lines) in dividing WT, Klp4 $\Delta$, Dnm1 $\Delta$ and Mmb1 $\Delta$ cells. The shaded region represents the standard deviation. D, Plot of total curvature of the cell in dividing WT, Klp4 $\Delta$, Dnm1 $\Delta$ and Mmb1 $\Delta$ cells. The asterisks $\left(^{* \star \star *}\right)$ represent $\mathrm{p}<10^{-4}$, Kruskal-Wallis test for non-parametric data.

with fragmented mitochondria producing increased ROS and 165 fused mitochondria producing reduced ROS (21). However, 166 we did not see a difference in mitochondrial ROS in mutants 167 with altered mitochondrial morphology (17). So too, trans- 168 formation of Dnm1 $\Delta$ cells with Dnm1 restored mitochon- 169 drial form (17) and also symmetry in daughter cell length 170 and area during division (Fig. S1C, D).

Mitochondrial morphology mutants exhibit cell polarity defects. In addition to changes in the cell division sym- ${ }_{174}$ metry, we observed that a large proportion of Dnm1 $\Delta$ and ${ }_{175}$ Mmb1 $\Delta$ cells showed aberrant polarity. Typical WT fission ${ }_{176}$ yeast cells have two defined poles, and therefore two areas ${ }_{177}$ of high curvature along the perimeter of the cell (Fig. 2A, $\mathrm{B}_{178}$ and C). However, interphase and diving cells in both Dnm1 $\Delta_{179}$ and Mmb1 $\Delta$ backgrounds exhibited aberrant polarities (Fig. ${ }_{180}$ 2A,2C, S1E). While most Dnm1 $\Delta$ and Mmb1 $\Delta$ cells con- ${ }_{181}$ tained two defined poles, several cells exhibited additional ${ }_{182}$ poles, as evinced by the higher standard deviation in the po- ${ }_{183}$ sition of the poles in these cells (Fig. 2C, S1E). Klp4 $\Delta$ and ${ }_{184}$ Pom $1 \Delta$ cells showed a large variation in the pole location due ${ }_{185}$ to the severe polarity defects that are expected in these mu- ${ }_{186}$ tants (Fig. 2A, D, S1F). We further measured the polarity in ${ }_{187}$ WT and mutant cells by summing the total curvature of cells ${ }_{188}$ - in this scenario, cells with enhanced polarity defects would ${ }_{189}$ have a net curvature that is higher than WT cells. Indeed, we ${ }_{190}$ observed that both Dnm1 $\Delta$ and Mmb1 $\Delta$ cells had total cur- 191 vatures that were significantly higher than that of WT cells ${ }_{192}$ (Fig. 2D). Again, Klp $4 \Delta$ and Pom1 $\Delta$ cells served as posi- ${ }_{193}$ tive controls (Fig. 2D, S1F), and reintroduction of Dnm1 to Dnm1 $\Delta$ cells restored normal polarity (Fig. S1F). In contrast 194 to the trends seen for cell division asymmetry, we noted that 195 $\mathrm{Klp} 5 / 6 \Delta$ cells did not exhibit polarity defects, whereas $r h o^{0}{ }_{196}$ cells did (Fig. S1F).

Microtubule dynamics are altered in mitochondrial ${ }_{199}$ morphology mutants. Nuclear positioning in S. pombe is 200 effected by pushing forces of growing MTs against the cell ${ }_{201}$ poles (4). Since there are around equal numbers of MT bundles on either side, the nucleus largely remains in the centre of the cell and this central location of the nucleus is essential in dictating the future cell division plane. Fission yeast MT mutants, such as Klp4 $\Delta$ and Klp5/6 $\Delta$, have altered MT dynamics, and therefore mis-center the nucleus, leading to a large proportion of asymmetrically dividing cells (Fig. 1C, D, S1C, D). We asked whether Dnm1 $\Delta$ and Mmb1 $\Delta$ cells displayed asymmetry in cell division due to altered MT dynamics. Mmb1 $\Delta$ cells have been described to have more dynamic MTs than WT cells (16). So too, Dnm1 $\Delta$ cells required a higher concentration of the MT-depolymerising drug TBZ to completely abrogate MTs (15), indicating higher MT stability. We measured the MT polymerisation rate, depolymerisation rate and MT elongation time in WT, Klp4 $\Delta$, Dnm $1 \Delta$ and Mmb1 $\Delta$ cells (Fig. 3A), and observed that MTs in Dnm1 $\Delta$ cells had an unaltered polymerisation rate (Fig. 3B), reduced depolymerisation rate (Fig. 3C) and increased elongation time (reduced catastrophe frequency) compared to WT cells (Fig. 3D). On the other hand, Mmb1 $\Delta$ cells had MTs with increased depolymerisation rate (Fig. 3C). As expected, Klp $4 \Delta$ cells exhibited reduced MT depolymerisation rate and polymerisation rate compared to WT cells (Fig. 3A, $\mathrm{B}$ and $\mathrm{C}$ ). These results indicated that the association of mitochondria with MTs enhanced MT stability, whereas the lack of association reduced MT stability. We confirmed that these results were not an artefact of the levels of tubulin expression in these cells by comparing the total intensity of tubulin among the strains employed (Fig S2A).

The nucleus is highly dynamic in mitochondrial morphology mutants. Since the nuclear position prior to onset of mitosis determines the future site of division (4), we set out to ask if the altered MT dynamics in the mitochondrial morphology mutants changed the nuclear dynamics in these cells. We observed that unlike WT cells, the nucleus was highly dynamic in both Dnm1 $\Delta$ and Mmb1 $\Delta$ cells (Fig. 4A, Video $\mathrm{S} 2$ ). As a result, the excursions of the nucleus from the 
A
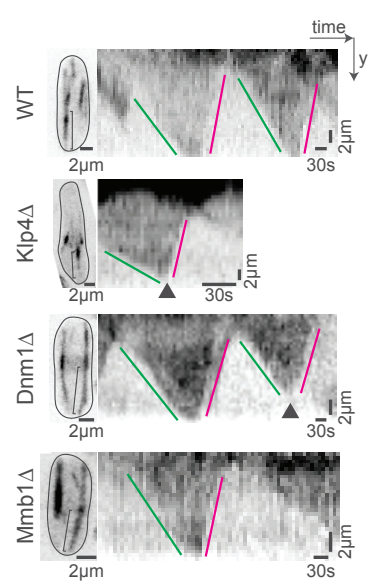

C

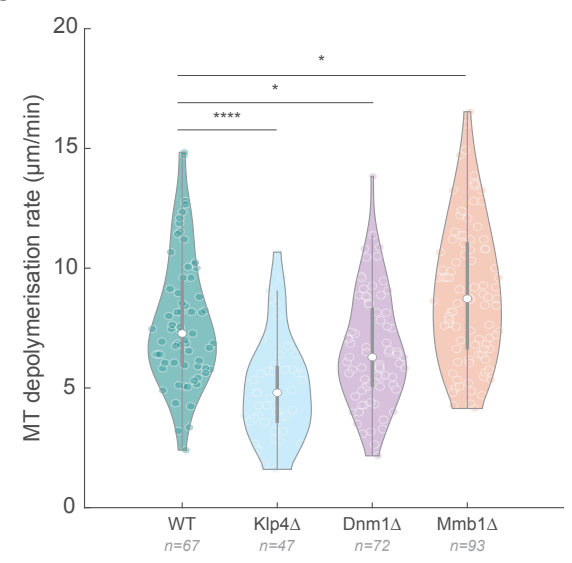

B
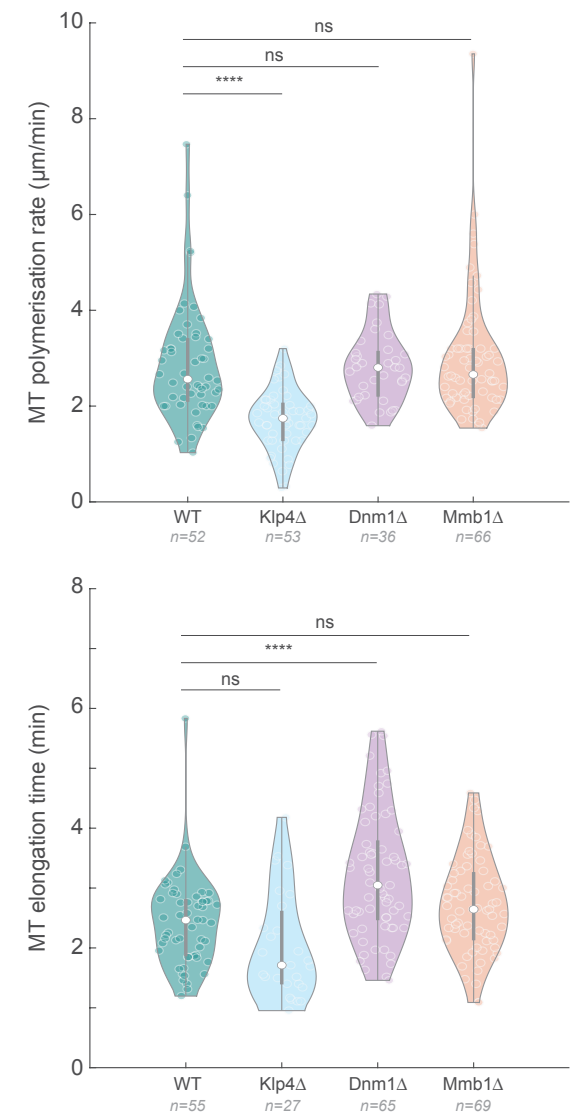

Fig. 3. MT depolymerisation rate is aberrant in Dnm1 $\Delta$ and Mmb1 $\Delta$ cells. A, Maximum intensity-projected images (left) of MTs from the first frame of time-lapse videos of representative WT, Klp4 $\Delta$, Dnm1 $\Delta$ and Mmb1 $\Delta$ cells (strains VA112, G5B, VA110 and VA113, see Table S1), and the corresponding kymographs (right) of the MTs indicated with the square brace. Green lines indicate MT polymerisation, magenta lines indicate MT depolymerisation and the arrowheads point to catastrophe events. B, Plot of MT polymerisation rates in WT, Klp4 $\Delta$, Dnm1 $\Delta$ and Mmb1 $\Delta$ cells (mean \pm S.D.: $2.9 \pm 1.2,1.7 \pm 0.6,2.8197 \pm 0.7$, and $3.0 \pm 1.3 \mu$ m/min respectively). C, Plot of MT depolymerisation rates in WT, Klp4 $\Delta$, Dnm1 $\Delta$ and Mmb1 $\Delta$ cells (mean \pm S.D.: $7.8 \pm 2.7,5.0 \pm 2.1,6.7 \pm 2.3$, and 9.0 $\pm 2.9 \mu$ m/min respectively). D, Plot of MT elongation times in WT, Klp4 $\Delta$, Dnm1 $\Delta$ and Mmb1 $\Delta$ cells (mean \pm S.D.: $2.4 \pm 0.7,2.0 \pm 0.9,3.2 \pm 1.1$, and $2.7 \pm 0.8$ min respectively). The reciprocal of the MT elongation time gives the MT catastrophe rate. In B, C and D, the asterisks represent significance $\left({ }^{* \star \star}=p<10^{-4}\right.$ and * $=p<11 \times 10^{-3}$ respectively), and 'ns' indicates no significant difference using Kruskal-Wallis test for non-parametric data and ordinary one-way ANOVA for parametric data.

cell centre were significantly higher in $\operatorname{Dnm} 1 \Delta$ and $\operatorname{Mmb} 1 \Delta_{223}$ cells than in WT cells (Fig. 4B). We confirmed that the nu- 224 cleus moved more as a result of the altered MT dynamics by 225 visualising the nuclear dynamics in cells devoid of MT (Fig. 226 S2B). As expected, we measured negligible movement of the 227 nucleus in the absence of MTs. So too, the short MTs in ${ }_{228}$ Klp4 $\Delta$ cells typically do not contact the cell end $(17,18),{ }_{229}$ and therefore does not result in a pushing force to move the 230 nucleus. This was reflected in the reduced movement of the 231 nucleus (Fig. 4A, Video S2), and increased distance of the 232 Klp4 $\Delta$ nuclei from the cell centre (Fig. 4B). Occasionally, 233 we observed Dnm1 $\Delta$ and Mmb1 $\Delta$ cells that had inherited ${ }_{234}$ few or no mitochondria from the mother cell. Remarkably, 235 the nuclei in these cells exhibited dramatic movements, reit- 236 erating that MT instability could be effected by lack of mito- 237 chondrial attachment (Fig. S2C, D, Video S3). Additionally, 238 in these cells that inherited few or no mitochondria, the net 239 cytoplasmic viscosity was likely reduced due to the lack of 240 an organelle that occupies upto $35 \%$ of the yeast cell vol- 241 ume (22), which could have also contributed to the increased 242 movement of the nucleus.
Microtuble pivoting is enhanced in mitochondrial morphology mutants. We next turned our attention back to the polarity defect phenotype that we observed in the mitochondrial morphology mutants. Polarity in fission yeast is a complex process that is specified by the delivery of growth factors to the cell poles primarily by the activity of MT-based kinesin-like proteins and +TIP binding proteins $(7,8)$. Therefore, in typical WT cells with MTs oriented and growing along the long axis of the cell, the cell only grows along this axis. We hypothesised that in the mitochondrial morphology mutants the enhanced or absence of attachment of mitochondria to MTs resulted in altered MT pivoting. This pivoting could lead to the deposition of growth factors in locations other than along the long axis of the cell. To test this, we visualised and measured the angle of MT bundles between one frame and the next in time-lapse videos of fluorescentlylabeled MTs in WT, Klp4 $\Delta$, Dnm $1 \Delta$ and Mmb1 $\Delta$ cells (Fig. 5A, B, Video S4). We observed that while Klp4 $\Delta$ cells that are known to show polarity defects also exhibited an increased median in pivoting of MTs between frames, Dnm1 $\Delta$ and Mmb1 $\Delta$ cells did not. However, both mitochondrial 
A

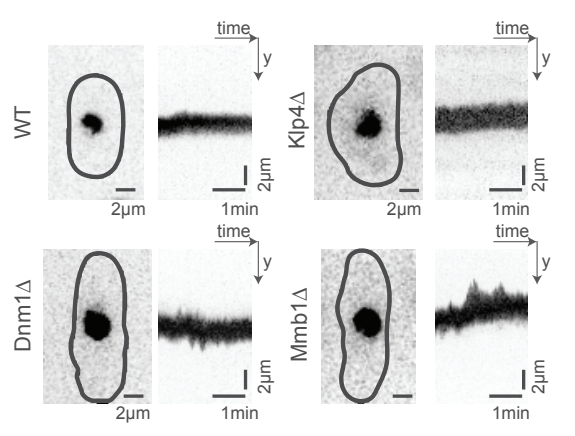

B

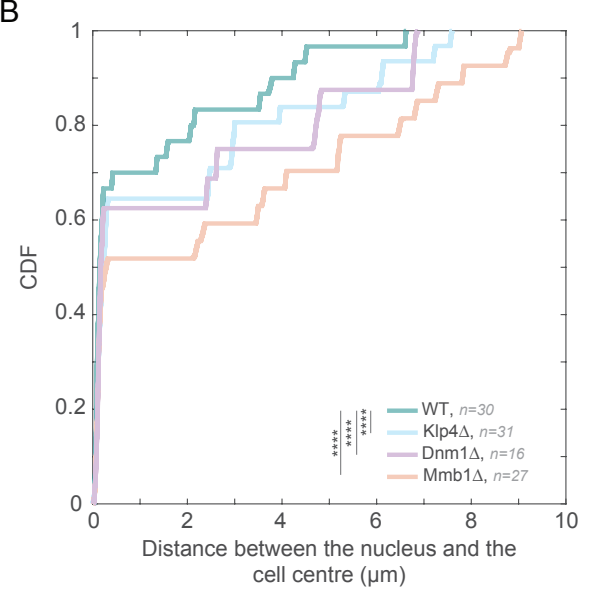

Fig. 4. Dnm1 $\Delta$ and Mmb1 $\Delta$ cells exhibit enhanced nuclear movement. A, Maximum intensity-projected images (left) of the nucleus from the first frame of time-lapse videos of representative WT, Klp4 $\Delta$, Dnm1 $\Delta$ and Mmb1 $\Delta$ cells (strains VA102, VA111, VA103 and VA104, see Table S1), and the corresponding kymographs (right) of the nuclear movement. B, Cumulative density function (CDF) of the distance of the nucleus from the cell centre for each time point of the time-lapse videos of nuclei in WT, $\mathrm{KIp} 4 \Delta$, Dnm1 $\Delta$ and Mmb1 $\Delta$ cells. The asterisks $\left({ }^{* * *}\right)$ represent $\mathrm{p}<10^{-4}$, Kruskal-Wallis test for non-parametric data.

morphology mutants had much higher deviations in MT piv- 277 oting than WT (Fig. 5B). Shorter MTs typically pivot more than longer MTs (23). However, when we visualised individ- ${ }^{278}$ ual MTs pivoting during their lifetime (growth or shrinkage), ${ }^{279}$ we observed that the mutants exhibited angles that were fur- ${ }^{280}$ ther from the long axis of the cell than WT (Fig. 5C). We ${ }^{281}$ next asked if MTs of comparable lengths pivoted more in the ${ }^{282}$ mitochondrial morphology mutants than WT cells. Indeed, at ${ }^{283}$ shorter MT lengths, Mmb1 $\Delta$ cells pivoted much more than ${ }^{284}$ WT cells (Fig. 5C), whereas Dnm1 $\Delta$ cells displayed slightly ${ }^{285}$ higher pivoting angles than WT cells at longer MT length ${ }^{286}$ (Fig. 5D). These difference in MT pivoting in the mutants ${ }^{287}$ likely resulted in the polarity defects we observed.

Mitochondrial partitioning is asymmetric in mitochon- 294 drial morphology mutants. Finally, we probed the conse- 295 quence of asymmetric division of mutant cells on the par- 296 titioning of mitochondria. Mitochondria undergo indepen- 297 dent segregation in fission yeast, with cell division symme- 298 try aiding the equitable partitioning of mitochondria between 299 daughter cells (17). We measured the amount of mitochon- 300 dria in dividing WT and mutant cells (Fig. 6A), and observed 301 that mitochondria were partitioned in proportion to the cell 302 area, indicating that independent segregation was still likely ${ }_{303}$ active in the mutants (Fig. 6B). However, since a signifi- 304 cant proportion of cells underwent asymmetric division in the 305 mutants, mitochondria were also partitioned unequally be- 306 tween daughter cells (Fig. 6C). We tested the outcome of 307 such asymmetric partitioning of mitochondria in a represen- 308 tative Dnm1 $\Delta$ cell that underwent asymmetric cell division 309 and observed that the smaller daughter cell grew slower than ${ }_{310}$ the larger daughter cell upon division, likely due to inheri- 311 tance of a smaller volume of mitochondria (Fig. S3, Video 312 S5).

\section{Discussion}

The interplay of mitochondria and MTs has been implicated in maintaining cellular homeostasis. Here, we first identified that alteration of mitochondrial form and thereby, attachment of mitochondria to MTs resulted in higher rates of incidence of asymmetry in typically symmetrically-dividing fission yeast cells. We showed that this asymmetry resulted from changes in MT depolymerisation rate and catastrophe frequency when the association of mitochondria to MTs was either enhanced or absent compared to WT cells. In metazoans, mitochondria rely on microtubules for their transport and positioning (24). Further, MTs in metazoans have been demonstrated to effect changes in gene expression owing to their link with the nuclear membrane via the LINC (linker of nucleoskeleton and cytoskeleton) complex (25). It would be interesting to see if a change in mitochondrial form or attachment to MTs has a similar effect on MT dynamics, and therefore cell fate in metazoans.

In addition to altered MT dynamics, cells with longer or shorter mitochondria also exhibited changes in MT pivoting. Shorter MTs pivot more due to the reduced force required for their pivoting (23). Here, we observed that MTs pivoted more in Mmb1 $\Delta$ cells, where mitochondria are detached from MTs - in WT cells, the attachment of mitochondria to MTs likely presented a barrier to pivoting, which was removed in Mmb1 $\Delta$ cells. MTs would therefore likely be present in orientations other than the long axis, leading to growth along these aberrant orientations. On the other hand, we also observed that MTs (especially longer MTs) in Dnm1 $\Delta$ cells also showed a slightly higher pivoting. Combined with the reduced catastrophe frequency in Dnm1 $\Delta$ MTs, this likely lead to deposition of growth factors at locations other than the cell poles in a fraction of cells.

The perturbation of MT dynamics in fission yeast mutants with altered mitochondrial form resulted in increased nuclear movements, which gave rise to nuclear positioning that was offset from the cell centre. Since fission yeast relies 
A
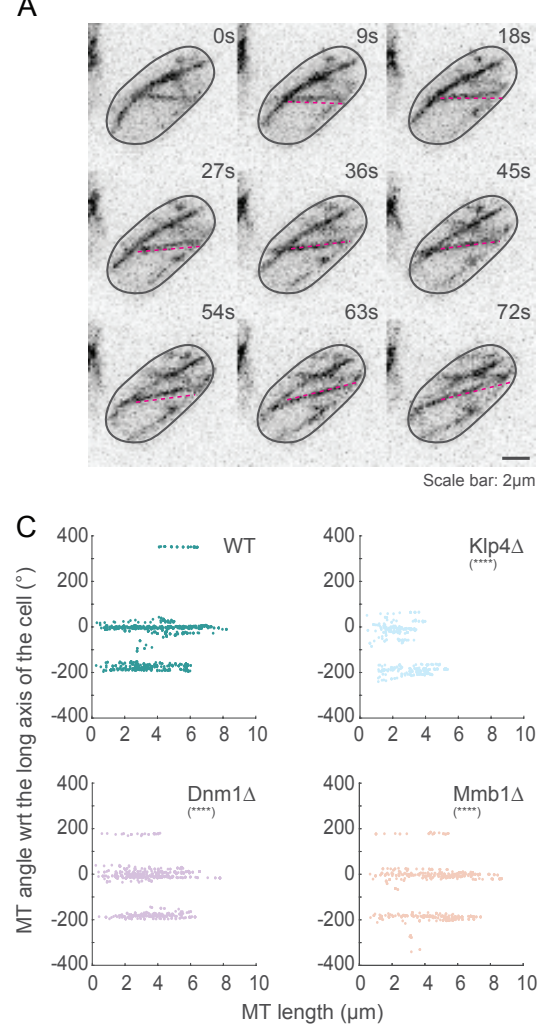

B

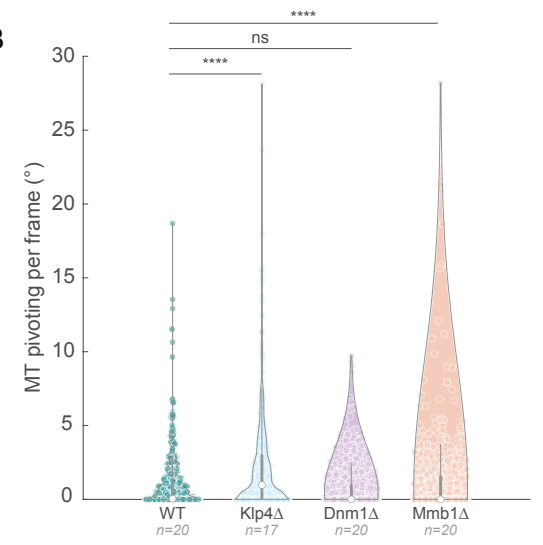

$\mathrm{D}$

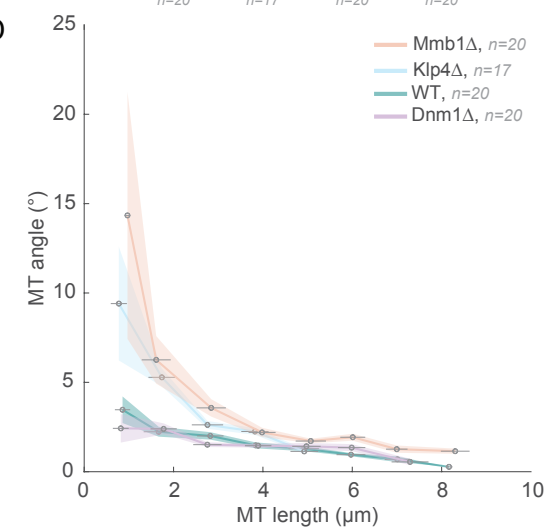

Fig. 5. MTs undergo increased pivoting in Dnm1 $\Delta$ and Mmb1 $\Delta$ cells. A, Montage of maximum intensity-projected images of MTs in a representative WT cell (strain VA112, see Table S1). The position of the MT at time 0s is indicated with the dashed magenta line in the image of time 9s, that in time 9s with the dashed magenta line in the image of time $18 \mathrm{~s}$ and so on. B, Plot of MT pivoting per frame in WT, Klp4 $\Delta$, Dnm1 $\Delta$ and Mmb1 $\Delta$ cells. While the data are not significantly different for Dnm1 $\Delta$ and Mmb1 $\Delta$ cells compared to WT, the spread of the data is much higher in Dnm1 $\Delta$ and Mmb1 $\Delta$ cells. C, Plot of MT angle with respect to the long axis of the cell versus MT length in WT, KIp4 $\Delta$, Dnm1 $\Delta$ and Mmb1 $\Delta$ cells. D, Plot of the data in B binned by MT length. In $\mathbf{B}$ and $\mathbf{C}$, the asterisks ( $\left.{ }^{* * *}\right)$ represent $\mathbf{p}<10^{-8}$ and 'ns' indicates no significant difference compared to WT using Levene's test for equal variance for non-parametric data.

on the nuclear position prior to mitosis to dictate the eventual ${ }_{3 з}$ cell division plane, mutants with altered mitochondrial form ${ }_{339}$ exhibited more instances of asymmetric cell division compared to WT cells.

Fission yeast as well as other metazoans have been documented to follow independent segregation to partition mito- ${ }^{34}$ chondria among daughter cells during mitosis $(17,26)$. Inde- ${ }^{342}$ pendent segregation relies on the presence of a large 'copy ${ }^{34}$ number' of mitochondria present in the mother cell so as ${ }^{34}$ to reduce the partitioning error (27). Given large enough ${ }^{345}$ copy numbers of mitochondria, positioning the division plane ${ }^{346}$ roughly at the cell centre ensures equitable distribution of ${ }^{347}$ mitochondria in daughter cells. In Mmb1 $\Delta$ and Dnm1 $\Delta^{348}$ cells, due to the asymmetry observed in a significant propor- ${ }^{349}$ tion of cells, mitochondrial partitioning between the daugh- ${ }^{350}$ ters though equitable, resulted in cells with very few mito- ${ }^{351}$ chondria compared to the rest of the population. These cells ${ }^{352}$ that contained fewer mitochondria grew slower, and therefore would likely be out-competed by other cells. However, because the reduction in mitochondria resulted from altered MT dynamics, asymmetric cell division and thereby again daugh- 354 ter cells with fewer mitochondria, would persist in future di- 355 vision cycles. Dnm1 $\Delta$ cells have previously been shown to 356 have retarded growth rates (28), which could be attributed to 357 the unequal partitioning of mitochondria following asymmetric cell division in a significant proportion of these cells.

\section{Conclusion}

In conclusion, MT dynamics and mitochondrial form and attachment were found to be fine-tuned to be in a 'Goldilocks zone' in fission yeast whereby symmetric cell division could be achieved and polarity could be maintained. Any deviation from this narrow range resulted in asymmetric cell division and polarity defects. Additionally, cellular homeostasis relied on the feedback between MTs and mitochondria, with the mitochondria dictating its own partitioning via changes in its form. In future, it will be interesting to understand the fate of cells that inherited fewer mitochondria, and if similar feedback mechanisms exist between the cytoskeleton and other intracellular compartments.

\section{Materials and Methods}

Strains and media. The fission yeast strains used in this study are listed in Table S1. All the strains were grown in yeast extract medium or Edinburgh minimal medium (EMM) with appropriate supplements at a temperature of $30^{\circ} \mathrm{C}(1)$. 

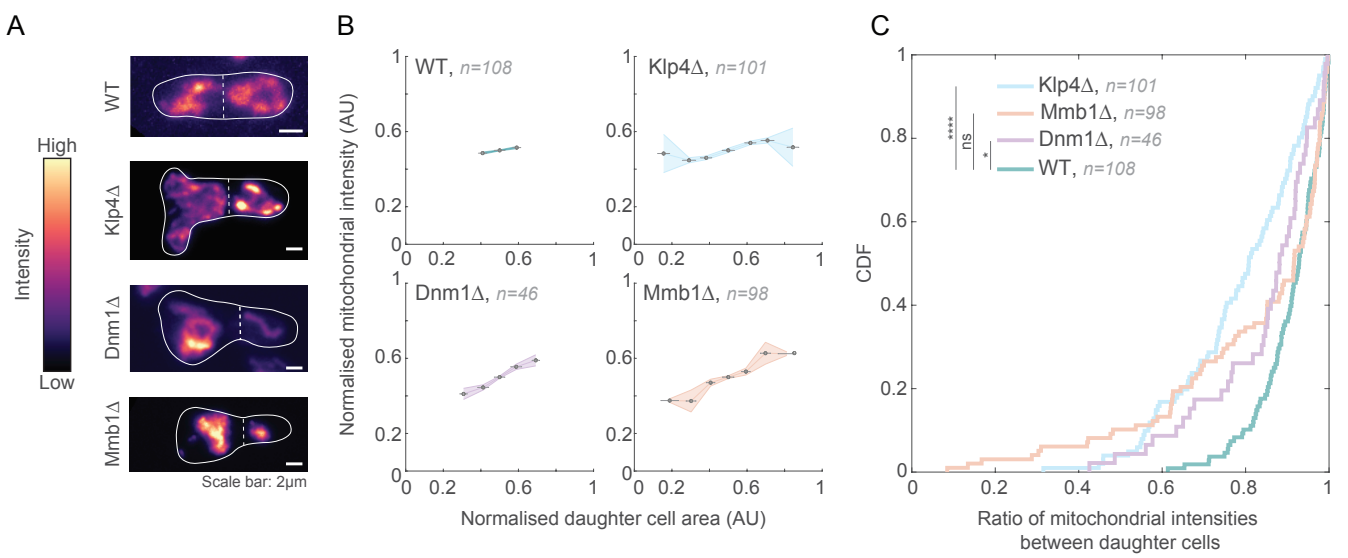

Fig. 6. Mitochondria are asymmetrically partitioned in Dnm1 $\Delta$ and Mmb1 $\Delta$ cells. A, Maximum intensity-projected images of mitochondria in WT, Klp4 $\Delta$, Dnm1 $\Delta$ and Mmb1 $\Delta$ cells (strains KI001, G5B, VA069 and PT2244, see Table S1). Warmer colours indicate higher intensities. The cell outlines are indicated with the solid white line and the septum between the daughter cells is the dashed white line. B, Plots of normalised mitochondrial intensity (sum intensity) vs. normalised cell area in WT, Klp4 $\Delta$, Dnm $1 \Delta$ and Mmb1 $\Delta$ cells. C, CDF of ratio of mitochondrial intensities between daughter cells in WT, Klp4 $\Delta$, Dnm1 $\Delta$ and Mmb1 $\Delta$ cells. The asterisks represent significance $(* \star \star *=$ $\mathrm{p}<10^{-4}$ and ${ }^{*}=\mathrm{p}<3 \times 10^{-2}$ respectively) and 'ns' indicates no significant difference using Kruskal-Wallis Test for non-parametric data.

Construction of strains. Strain VA064 was constructed by 394 transforming Dnm1 $\Delta$ with pREP41-Dnm1 (Dnm1 untagged 395 plasmid). Similarly, strain VA102 was constructed by cross- 396 ing PT1650 (h+ cox4-GFP:leu1 ade6-M210 ura4-D18; see ${ }_{397}$ Table S1) with JCF4627 (h- ade6-M210 leu1-32 ura4-D18 his3-D1 hht1-mRFP-hygMX6; see Table S1) while strain 398 VA103 was constructed by crossing VA077 (h- dnm1::kanr ${ }^{999}$ leu1-32ade-(ura+)cox4-GFP:leu1 ade6-M210 leu1-32 ura4- 400 D18; see Table S1) with VA101 (h+ hht1-mRFP-hygMX6 401 cox4-GFP:leu1 ade6-M210 leu1-32 ura4-D18; see Table 402 S1). Strain VA104 was constructed by crossing VA080 403 (h- mmb1 $\Delta$ :Kanr cox4-GFP:leu2 mCherry-atb2:Hygr ade6- 404 m210 leu1-32 ura4-d18; see Table S1) with VA101 $(\mathrm{h}+\mathrm{hht1}-405$ mRFP-hygMX6 cox4-GFP:leu1 ade6-M210 leu1-32 ura4- 406 D18; see Table S1). Strain VA110 was constructed by 407 crossing VA109 (h+ dnm1 $\Delta:: k a n r$ leu1-32ade-(ura+) ura4- 408 $\Delta 18$ leu1::GFP-atb2+:ura4+; see Table S1) with JCF4627 409 (h- ade6-M210 leu1-32 ura4-D18 his3-D1 hht1-mRFP- 410 hygMX6). Strain VA111 was constructed by crossing VA102 411 (h- hht1-mRFP-hygMX6 cox4-GFP:leu1 ade6-M210 leu1- 412 32 ura4-D18; see Table S1) with MCI438 ( $h+$ tea2d:his3 ade6 413 leu1-32 ura4-D18 his3-D1; see Table S1). Strain VA112 414 was constructed by crossing JCF4627 (h- ade6-M210 leu1- 415 32 ura4-D18 his3-D1 hht1-mRFP-hygMX6; see Table S1) 416 with VA106 (h+ ura4- $\Delta 18$ leu1::GFP-atb2+:ura4+; see Ta- 417 ble S1). Strain VA113 was constructed by crossing VA112 418 (h+ hht1-mRFP-hygMX6 ura4- $\Delta 18$ leu1::GFP-atb2+:ura4+ 419 ade6-M210 leu1-32 his3-D1; see Table S1) with VA078 ( $\mathrm{h}+420$ mmb1 $\Delta$ :Kanr; see Table S1).

Plasmid transformation Transformation of strains was 4 carried out using the improved protocol for rapid transfor- 424 mation of fission yeast as described previously (17).

Preparation of yeast for imaging. For imaging, fission 426 yeast cells were grown overnight in a shaking incubator at ${ }_{227}$ $30^{\circ} \mathrm{C}$. The following day the cells were washed once with ${ }_{428}$ distilled water and thrice with EMM. The cells were then ${ }_{429}$ allowed to adhere on lectin-coated (Sigma-Aldrich, catalog number L2380) 35-mm confocal dishes (SPL Life Sciences, cat. number 100350) for $20 \mathrm{~min}$. Unattached cells were removed by washing with EMM.

Microscopy. Confocal microscopy was carried out in Fig. 1A, 2A, 6A, S1B, S2C and S3A using the InCell Analyzer6000 (GE Healthcare) with a 60x air objective 0.95 numerical aperture (NA) objective fitted with an sCMOS camera. For GFP and RFP imaging, 488 and $561 \mathrm{~nm}$ laser lines and $525 / 20$ and $605 / 52 \mathrm{~nm}$ bandpass emission filters, respectively, were used. Spinning disk confocal microscopy was carried out in Fig. 3A, 4A, 5A and S1A using the Eclipse Ti2-E (Nikon) with a $100 \times$ oil-immersion 1.49 NA objective fitted with an EMCCD camera (iXon Ultra-897; Andor). For GFP and RFP imaging, 488 and $561 \mathrm{~nm}$ laser lines (Toptica) and 525/20 and 605/52 $\mathrm{nm}$ bandpass emission filters, respectively, were used.

MT polymerisation, depolymerisation rates and MT pivoting in Fig. 3B and 5A were obtained by imaging Z-stacks (7 slices with step size $1 \mu \mathrm{m}$ ) acquired every $3 \mathrm{~s}$ for $5 \mathrm{~min}$. MT elongation times in Fig. 3D were imaged using Z-stacks (7 slices with step size $1 \mu \mathrm{m}$ ) acquired every $7 \mathrm{~s}$ for $10 \mathrm{~min}$. Short term nuclear dynamics in Fig. 4A were imaged using Z-stacks (7 slices with step size $1 \mu \mathrm{m}$ ) acquired every $20 \mathrm{~s}$ for 20 min while long-term nuclear dynamics in Fig. S2C were imaged using Z-stacks (5 slices with step size $0.5 \mu \mathrm{m}$ ) every 15 minutes for 12 hours. MT depolymerisation in Fig. S1A was observed in time-lapse movies containing Z-stacks (5 slices with step size $0.5 \mu \mathrm{m}$ ) acquired every $12.5 \mathrm{~s}$ for 20 min. The growth rates of divided daughter cells in Fig. S3A and Fig. S3B was imaged with Z-stacks (5 slices with step size $0.5 \mu \mathrm{m}$ ) every $15 \mathrm{~min}$ for $12 \mathrm{~h}$.

Image and data analysis. Images were analysed using Fiji/ImageJ (29). The curvatures in Fig. 2C were measured by drawing an ROI around the perimeter of the cell and using the curvature_radius.bsh script for ImageJ (O. Burri, EPFL) 
bioRxiv preprint doi: https://doi.org/10.1101/2021.12.19.473396; this version posted December 20, 2021. The copyright holder for this preprint (which was not certified by peer review) is the author/funder, who has granted bioRxiv a license to display the preprint in perpetuity. It is made available under aCC-BY-NC-ND 4.0 International license.

that takes a selection and computes the radius of curvature 479 progressively for the whole perimeter of the shape. The curvature values and cell lengths were normalised in order to ${ }_{48}^{480}$ plot the mean and standard deviations. The total curvature ${ }^{482}$ was obtained by computing the sum of curvature values for $a^{483}$ cell.

The MT polymerisation and depolymerisation rates were ${ }_{487}^{486}$ obtained by measuring the angle of the slopes $(\theta)$ from ky- ${ }^{488}$ mographs generated by drawing a line along a growing or ${ }_{490}^{489}$ shrinking MT and using the following formula:

$$
\frac{x}{y}=\tan \theta \times \frac{\text { pixel size }}{\text { time interval }(\mathrm{sec})} \times 60
$$

Where:

\section{$\mathrm{x}$ : is the MT length in $\mu \mathrm{m}$}

$y:$ is the time in $\min$

The MT elongation time was calculated from the kymo- ${ }^{500}$ graph by measuring the time from the onset of polymerisa- 508 tion to a catastrophe event. The rate of catastrophe was ob- ${ }_{510}^{509}$ tained from the reciprocal of the mean elongation time. The 511 nuclear dynamics were obtained by thresholding the nucleus ${ }_{513}^{512}$ from time-lapse videos in ImageJ to obtain the nuclear cen- 514 troid, and drawing an ROI around the cell perimeter to get ${ }_{516}^{515}$ the cell centroid. Then the euclidian distance between the 2517 centroids was then calculated.

The nuclear velocity in Fig. S2D by measuring the eu- 520 clidean distance between the nuclear positions in successive ${ }_{522}^{521}$ frames. MT pivoting was measured as the difference in the ${ }_{523}$ angle of the MT from one frame to another.

Statistics and plotting. Data were checked for normality using the chi2gof function in Matlab. Then, to test the sta- ${ }_{529}$ tistical significance of the difference between distributions ${ }_{531}^{530}$ we used ordinary one-way ANOVA for parametric data and ${ }_{532}^{531}$ Kruskal-Wallis test or Mann-Whitney test for non-parametric ${ }^{533}$ data. Levene's test for equal variances was used to compare ${ }_{535}^{534}$ the variances of non-parametric distributions in Fig. 5B and ${ }^{536}$ C. All plots were generated using Matlab (Mathworks Corp.). ${ }_{538}^{537}$ The figures were organised and prepared in Illustrator.

\section{Acknowledgements}

We thank Ananya Rajagopal for help with construction ${ }_{545}^{544}$ of strains; the High Content Imaging Facility, Centre for ${ }_{547}^{546}$ BioSystems Science and Engineering, Indian Institute of Sci- 548 ence for the use of the InCell 6000 and spinning disk con- ${ }_{550}^{549}$ focal microscopes; G. Redpath, N. Ul Fatima and A. Badri- 551 narayanan for comments on the manuscript; P. Delivani (Max ${ }_{553}^{552}$ Planck Institute of Molecular Cell Biology and Genetics, ${ }_{554}$ Dresden, Germany), F. Ishikawa (Kyoto University, Kyoto, ${ }_{556}^{555}$ Japan), M. Takaine (Gunma University, Gunma, Japan), I. ${ }^{557}$ Tolić (Ruđer Bošković Institute, Zagreb, Croatia), P. Tran ${ }_{559}^{558}$ (University of Pennsylvania, Philadelphia, PA), T.D. Fox 560 (Cornell University, Ithaca, NY), and National BioResource ${ }^{561}$ Project Japan for yeast strains and constructs.

\section{References}

1. Susan L. Forsburg and Nicholas Rhind. Basic methods for fission yeast. Yeast (Chichester, England), 23:173, 2 2006. ISSN 0749503X. doi: 10.1002/YEA.1347.

2. Matthieu Piel and Phong T. Tran. Cell shape and cell division in fission yeast minireview. Current biology : CB, 19:R823, 9 2009. ISSN 09609822. doi: 10.1016/J.CUB.2009.08.012.

3. I. Ju Lee, Valerie C. Coffman, and Jian Qiu Wu. Contractile-ring assembly in fission yeast cytokinesis: Recent advances and new perspectives. Cytoskeleton (Hoboken, N.J.), 69: 751, 10 2012. ISSN 19493584. doi: 10.1002/CM.21052.

4. P. T. Tran, L. Marsh, V. Doye, S. Inoué, and F. Chang. A mechanism for nuclear positioning in fission yeast based on microtubule pushing. The Journal of Cell Biology, 153:397, 42001. ISSN 00219525. doi: 10.1083/JCB.153.2.397.

5. Johanna L Höög, Cindi Schwartz, Angela T Noon, Eileen T O'Toole, David N Mastronarde, $\mathrm{J}$ Richard McIntosh, and Claude Antony. Organization of interphase microtubules in fission yeast analyzed by electron tomography. Developmental cell, 12(3):349-61, mar 2007. ISSN 1534-5807. doi: 10.1016/j.devcel.2007.01.020.

6. Rafael R Daga and Fred Chang. Dynamic positioning of the fission yeast cell division plane. Proceedings of the National Academy of Sciences of the United States of America, 102: 8228-32, 6 2005. ISSN 0027-8424. doi: 10.1073/pnas.0409021102.

7. Sophie G. Martin. Microtubule-dependent cell morphogenesis in the fission yeast. Trends in cell biology, 19(9):447-54, sep 2009. ISSN 1879-3088. doi: 10.1016/j.tcb.2009.06.003.

8. Sophie G. Martin and Robert a. Arkowitz. Cell polarization in budding and fission yeasts. FEMS Microbiology Reviews, 38(2):228-253, 2014. ISSN 01686445. doi: 10.1111/ 1574-6976.12055.

9. Benedikt Westermann. Molecular machinery of mitochondrial fusion and fission. The Journal of Biological Chemistry, 283(20):13501-5, may 2008. ISSN 0021-9258. doi: 10.1074/jbc.R800011200.

10. David C. Chan. Mitochondria: Dynamic Organelles in Disease, Aging, and Development. Cell, 125(7):1241-1252, 2006. ISSN 00928674. doi: 10.1016/j.cell.2006.06.010.

11. Prashant Mishra and David C. Chan. Mitochondrial dynamics and inheritance during cell division, development and disease. Nature Reviews Molecular Cell Biology, 15(10):634646, sep 2014. ISSN 1471-0072. doi: 10.1038/nrm3877.

12. Benedikt Westermann. Bioenergetic role of mitochondrial fusion and fission. Biochimica et Biophysica Acta (BBA) - Bioenergetics, 1817(10):1833-1838, oct 2012. ISSN 00052728. doi: 10.1016/j.bbabio.2012.02.033.

13. Elena Ingerman, Edward M Perkins, Michael Marino, Jason A Mears, J Michael McCaffery, Jenny E Hinshaw, and Jodi Nunnari. Dnm1 forms spirals that are structurally tailored to fit mitochondria. Journal of Cell Biology, 170(7):1021-1027, sep 2005. ISSN 1540-8140. doi: $10.1083 /$ jcb.200506078.

14. Jason A Mears, Laura L Lackner, Shunming Fang, Elena Ingerman, Jodi Nunnari, and Jenny E Hinshaw. Conformational changes in Dnm1 support a contractile mechanism for mitochondrial fission. Nature Structural \& Molecular Biology, 18(1):20-26, jan 2011. ISSN 1545-9993. doi: 10.1038/nsmb.1949.

15. Isabelle Jourdain, Yannick Gachet, and Jeremy S. Hyams. The dynamin related protein $\mathrm{dnm} 1$ fragments mitochondria in a microtubule-dependent manner during the fission yeast cell cycle. Cell motility and the cytoskeleton, 66:509-523, 8 2009. ISSN 1097-0169. doi: 10.1002/CM.20351.

16. Chuanhai Fu, Deeptee Jain, Judite Costa, Guilhem Velve-Casquillas, and Phong T. Tran. Mmb1p binds mitochondria to dynamic microtubules. Current Biology, 21:1431-1439, 9 2011. ISSN 09609822. doi: 10.1016/j.cub.2011.07.013.

17. K. Mehta, L.A. Chacko, M.K. Chug, S. Jhunjhunwala, and V. Ananthanarayanan. Association of mitochondria with microtubules inhibits mitochondrial fission by precluding assembly of the fission protein dnm1. Journal of Biological Chemistry, 294, 2019. ISSN 1083351X. doi: 10.1074/jbc.RA118.006799.

18. Heidi Browning, Jacqueline Hayles, Juan Mata, Lauren Aveline, Paul Nurse, and J. Richard McIntosh. Tea2p is a kinesin-like protein required to generate polarized growth in fission yeast. Journal of Cell Biology, 151(1):15-27, oct 2000. ISSN 00219525. doi: 10.1083/jcb. 151.1.15.

19. Jürg Bähler and John R. Pringle. Pom1p, a fission yeast protein kinase that provides positional information for both polarized growth and cytokinesis. Genes and Development, 12 (9):1356-1370, 1998. ISSN 08909369. doi: 10.1101/gad.12.9.1356.

20. P Haffter and T D Fox. Nuclear mutations in the petite-negative yeast Schizosaccharomyces pombe allow growth of cells lacking mitochondrial DNA. Genetics, 131(2):255-60, 1992.

21. O.Yu. Pletjushkina, K.G. Lyamzaev, E.N. Popova, O.K. Nepryakhina, O.Yu. Ivanova, L.V. Domnina, B.V. Chernyak, and V.P. Skulachev. Effect of oxidative stress on dynamics of mitochondrial reticulum. Biochimica et Biophysica Acta (BBA) - Bioenergetics, 1757(5-6): 518-524, may 2006. ISSN 00052728. doi: 10.1016/j.bbabio.2006.03.018.

22. Francesca Di Bartolomeo, Carl Malina, Kate Campbell, Maurizio Mormino, Johannes Fuchs, Egor Vorontsov, Claes M Gustafsson, and Jens Nielsen. Absolute yeast mitochondrial proteome quantification reveals trade-off between biosynthesis and energy generation during diauxic shift. Proceedings of the National Academy of Sciences, 117(13):7524-7535, mar 2020. ISSN 0027-8424. doi: 10.1073/pnas.1918216117.

23. Iana Kalinina, Amitabha Nandi, Petrina Delivani, Mariola R. Chacón, Anna H. Klemm, Damien Ramunno-Johnson, Alexander Krull, Benjamin Lindner, Nenad Pavin, and Iva M. Tolić-Nørrelykke. Pivoting of microtubules around the spindle pole accelerates kinetochore capture. Nature Cell Biology, 15(1):82-87, dec 2013. ISSN 14657392. doi: $10.1038 /$ ncb2640.

24. Mitali Shah, Leeba Ann Chacko, Joel P. Joseph, and Vaishnavi Ananthanarayanan. Mitochondrial dynamics, positioning and function mediated by cytoskeletal interactions. Cellular and Molecular Life Sciences, feb 2021. ISSN 14209071. doi: 10.1007/s00018-021-03762-5.

25. Mitra Shokrollahi and Karim Mekhail. Interphase microtubules in nuclear organization and genome maintenance. Trends in Cell Biology, 31(9):721-731, sep 2021. ISSN 0962-8924. doi: 10.1016/J.TCB.2021.03.014.

26. Elizabeth Lawrence and Craig Mandato. Mitochondrial inheritance is mediated by microtubules in mammalian cell division. Communicative \& integrative biology, 6(6):e27557, nov 2013. ISSN 1942-0889. doi: $10.4161 / \mathrm{cib} .27557$. 
bioRxiv preprint doi: https://doi.org/10.1101/2021.12.19.473396; this version posted December 20, 2021. The copyright holder for this preprint (which was not certified by peer review) is the author/funder, who has granted bioRxiv a license to display the preprint in perpetuity. It is made available under aCC-BY-NC-ND 4.0 International license.

$\begin{array}{lrl}564 & \text { 27. Dann Huh and Johan Paulsson. Random partitioning of molecules at cell division. Pro- } \\ 565 & \text { ceedings of the National Academy of Sciences of the United States of America, 108(36): } \\ 566 & \text { 15004-9, sep 2011. ISSN 1091-6490. doi: } 10.1073 / \text { pnas.1013171108. } \\ 567 & \text { 28. Fenfen Dong, Mengdan Zhu, Fan Zheng, and Chuanhai Fu. Mitochondrial fusion and fission } \\ 568 & \text { are required for proper mitochondrial function and cell proliferation in fission yeast. The } \\ 569 & \text { FEBS Journal, 8 2021. ISSN 1742-464X. doi: 10.1111/febs.16138. } \\ 570 & \text { 29. Johannes Schindelin, Ignacio Arganda-Carreras, Erwin Frise, Verena Kaynig, Mark Longair, } \\ 571 & \text { Tobias Pietzsch, Stephan Preibisch, Curtis Rueden, Stephan Saalfeld, Benjamin Schmid, } \\ 572 & \text { Jean-Yves Tinevez, Daniel James White, Volker Hartenstein, Kevin Eliceiri, Pavel Toman- } \\ 573 & \text { cak, and Albert Cardona. Fiji: an open-source platform for biological-image analysis. Nature } \\ 574 & \text { Methods, 9(7):676-682, jul 2012. ISSN 1548-7091. doi: 10.1038/nmeth.2019. }\end{array}$ 